\title{
Measure the Market Risk of the Options on Stock Indices Based on GJR-GARCH Model and Monte Carlo Simulation
}

\author{
Qiang Li Shaoxian Xia \\ Guizhou University of Finance and Economics, Guiyang, 550025, China
}

\begin{abstract}
This paper focused on the pricing models of the SSCI and SZCI options on stock indices and the volatility estimation of the GJR-GARCH models which its underlying assets portfolio obeys to. Combining with B-S pricing models and the mainstream approach for the market risk management -VaR,we study on the market risk of the options on stock indices using simulation and optimization. With the result of European put options on stock indices of SSCI and SZCI,95\%VaR can be measured by the Monte Carlo sampling and Latin Hypercubs sampling techniques approximation and the analysis formula separately. The result shows that these two different perspectives of sampling techniques can be applied to China's launch of stock index option risk measurement.
\end{abstract}

\section{Keywords}

Options on stock indices; B-S model; Mento Carlo simulation; Value at Risk

\section{基于 GJR-GARCH 模型和蒙特卡洛模拟 的股指期权的风险度量}

\author{
李强 夏少先 \\ 贵州财经大学，贵阳, 550025, 中国
}

摘要：本文侧重于对 GJR-GARCH 模型得到的波动率序列和股指期权进行研究，选取上证综 指和深证综指作为分析对象，根据 B-S 模型得到沪深综指的期权价格，以 B-S 模型期权价格 为基准, 采用数值方法对沪深综指的期权定价进行比较研究, 确认 B-S 模型得到的欧式看跌 
期权价格较为精确, 将欧式看跌期权作为输入变量, 进行蒙特卡洛和拉丁方抽样对比, 最后 基于 $\mathrm{VaR}$ 的 95\%置信水平对沪深综指期权的市场风险进行风险度量, 并得出有关结论。

关键词：股指期权；布莱克-斯科尔斯模型；蒙特卡洛模拟；在险价值

\section{1.引言}

随着金融创新和世界经济一体化 进程的加快，国际金融市场巨幅波动， 我国中小投资者迫切需要一种能够有 效规避风险、实现资产保值的金融工 具。作为新兴发展的衍生工具, 股指期 权利用交易双方风险收益机制的不对 称和权责的不平等，可以在期货市场上 规避股指期货风险，为中小投资者在限 定风险情况下追求更高的收益提供了 一种可能路径。在防范和化解金融风险 方面, Black-scholes 定价模型基于有效 市场等一系列假设条件下，推导出基于 不支付红利股票的任何衍生证券的价 格必须满足的微分方程。Penza 和 Bansal $^{[1]}$ (2001) 运用 VaR 方法对金融衍 生工具定价方面做了简要阐述。Cormac Butler $^{[2]}$ (2002) 依据固定收益证券定价 的持续期一凸性方法, 将 VaR 模型应用 于非线性期权的定价方法进行了深入 研究。国内文献主要集中对期权定价模 型的改进及 VaR 的概述与测算上, 对运 用 VaR 测算期权风险方面的文献相对较 少。杨春鹏等 ${ }^{[3]}$ (1999) 通过一定的假 设条件，给出了度量一类非对称型金融 衍生工具市场风险的 VaR 的解析式。张

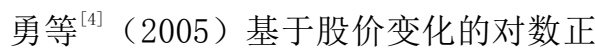
态模型, 给出了欧式看涨看跌期权的 VaR 计算方法。从上述文献中可以得知
运用 VaR 方法对中国股指期权的前瞻性 研究相对缺乏, 特别是利用拉丁方抽样 技术对中国股指期权进行风险度量, 就 笔者掌握的文献而言，比较罕见。

\section{2. 模型分析}

\section{1. AR-GJR-GARCH 模型}

考虑到沪深综指的收益率存在厚 尾、非对称等特征, 本文采用 AR (1) 来描述其条件均值, GARCH $(1,1)$ 来度 量其条件方差，刻画波动率的集聚效 应。同时应用 GJR-GARCH 模型描述非对 称的杜杆效应, 并对波动率进行建模。 AR (1)-GJR-GARCH $(1,1)$ 波动模型如下: $\left\{\begin{array}{l}R_{t}=\mu+\varphi R_{t-1}+\varepsilon_{t} \\ \varepsilon_{t}=\sigma_{t} Z_{t} \\ \sigma_{t}^{2}=\omega+\alpha \varepsilon_{t-1}^{2}+\gamma I_{t-1} \varepsilon_{t-1}^{2}+\beta \sigma_{t-1}^{2}\end{array}\right.$

其中: $\omega>0, \alpha \geq 0, \beta \geq 0, \alpha+\beta<1, R_{t}=\ln P_{t}-\ln P_{t-1}$ 为实际收益率, $\mu_{i}$ 为预期收益率, $\sigma_{t}$ 为 $t$ 日收益的波动率, $\gamma$ 为非对称杜杆系 数。 $\omega, \alpha, \mu, \beta, \gamma$ 为待估参数。

\subsection{B-S欧式期权定价模型}

Black和Scholes 基于无套利思想和 一系列严格假设, 推导出了期权定价方 程。B-S模型基本原理是基于中心极限 定理, 假定该标的资产组合价格变动过 程服从对数正态分布, 同时假定每个标 的资产价格服从几何布朗运动。利用 Delta对冲技术, 构建一个投资组合, 然 
后进行数学推导, 整理得到B-S方程:

$$
S r \frac{\partial f}{\partial S}+\frac{\partial f}{\partial t}+\frac{1}{2} S^{2} \sigma^{2} \frac{\partial^{2} f}{\partial S^{2}}=r f
$$

B-S 模型的重要性体现在对于任何 标的资产组合的动态方程必须满足此 方程。但有些期权定价难以找到精确的 解析公式, 在无封闭解的情形下, 期权 定价一般需要寻求数值解法。

\section{3. 数值方法一一二叉树模型}

Cox,Ross ${ }^{[5]}$ 提出的风险中性定价思 想是期权定价方法的理论基础。 Cox,Ross 和 Rubinstern ${ }^{[6]}$ (1979) 建立的 二叉树定价模型广泛应用于期权估价。 构建二叉树模型时，在时间区间 $\delta t$ 内, 使二叉树模型中的均值和方差与股票 价格的行为模式中推导出的均值和方 差相等, 以此建立方程组, 进而求出参 数 $p, u, d$ 。如考虑对一个不付红利股票 期权进行数值求解, 首先将期权的有效 期分为 $N$ 个时间间隔为 $\delta t$ 的区间。假 设在 $\delta t$ 时间内, 初始时刻股价 $S$ 分别以 概率 $p$ 和 $1-p$ 随机移动到期末的价 格, 若上涨时价格为 $u S$, 下降时价格为 $d S$ 。基于风险中性的假设, 经过 $\delta t$ 时间 股票价格的期望值为 $S e^{r \delta t}$, 其中 $r$ 为 无风险率。因而存在如下的约束方程:

$$
S e^{r t}=p S u+(1-p) S d \text { 或 } e^{r \delta t}=p u+(1-p) d
$$

由随机过程理论可知, 风险中性概 率下的方差应当是对应时间区间内的 方差 $\sigma^{2} \delta t$, 所以为满足方差的一致性, 随机变量 $Q$ 有如下的约束方程:

$$
E\left(Q^{2}\right)-[E(Q)]^{2}=E\left(S_{t+\delta t}\right)^{2}-E^{2}\left(S_{t+\delta t}\right)=\sigma^{2} S_{t}^{2} \delta t
$$

二叉树模型中，在 $t+\delta t$ 时刻，股价只 有两种可能: $u S$ 或 $d S$, 其对应的风险 中性概率分别为 $p$ 和 $1-p$ 。而
$E\left(S_{t+\delta t} / S_{t}\right)$ 为收益率的期望, 故(3)代入 (4)式可得:

$p u^{2}+(1-p) d^{2}-[p u+(1-p) d]^{2}=p u^{2}+(1-p) d^{2}-e^{2 r d}=\sigma^{2} \delta t$

对得到的两个约束方程整理后, 则

$\left\{\begin{array}{l}e^{r \delta t}=p u+(1-p) d \\ \sigma^{2} \delta t=p u^{2}+(1-p) d^{2}-e^{2 r \delta t}\end{array}\right.$

上述模型中, $r$ 由利率期限结构确 定, $\delta t$ 为人为设定, $\sigma^{2}$ 由股价历史数 据计算得出。显然上述模型两个方程却 有三个变量： $p, u, d$ 。因此需要增加一 个约束方程。Cox, Ross和Rubinstein通 过给出第三个约束条件 $u d=1$, 建立CRR 模型。其相应解为:

$p=(a-d) /(u-d), u=e^{\sigma \sqrt{\delta t}}, d=e^{-\sigma \sqrt{\delta t}}$. 其中, $a=e^{r \delta t}$ 而直接令第三个约束条件 $p=0.5$, 则为 $\mathrm{EQP}$ (等概率) 模型 ${ }^{[7]}$ 。

\section{4. 数值方法二---蒙特卡洛模拟方法}

蒙特卡洛方法是 Boyle $\mathrm{e}^{[8]}$ (1977) 引 入到金融分析领域并应用于期权定价 研究。它作为一种重要的求数值积分的 随机性方法, 用来模拟难以通过建立数 学模型进行定量分析的问题。其基本思 路是通过随机数模拟产生标的资产的 价格样本，然后计算期权损益的到期价 值，反复上述步骤，最后以期权损益的 平均到期价值作为期权价格。

\section{3. 采用VaR方法度量沪深股指期权风 险的前瞻研究}

随着中国金融市场的体系和环境 的完善，金融全球化是必然趋势，2010 年在推出股指期货之后，股指期权的推 出势在必行。本文选取上证综指和深证 综指作为期权的标的物，模拟设立股指 期权，对设立的股指期权的风险状况进 
行初步的研究, 为将来设立期权市场规 避风险提供参考。

沪深综指期权风险的模拟分析：本 文样本选取 1995 年 12 月 19 日至 2010 年 9 月 13 日的上证综指、深证综指的 日收盘指标作为期权的标的物, 样本容 量为 3567 组数据。收益率采取对数收 益率: $R_{t}=\ln \left(p_{t} / p_{t-1}\right)$ 。所有样本数据 均来源于国泰君安金融数据库 (CSMR)。 考虑分别设立两指数的欧式看涨和看 跌期权，指数的现值取 2010 年 9 月 13 日沪深综指收盘价, 即 $S_{1}=2688.32$, $S_{2}=1169.02$ 。无风险利率取 2010 年 1 月 1 日的一年期存款利率 $r=2.25 \%$ ，不 考虑指数的红利收益率。股指期权有效 期设为三个月, 分别选取整个样本波动 率均值和自 2010 年 9 月 13 日起倒推三 个月的条件波动率的均值进行比较, 沪 深综指波动率选取相对稳定的均值。具 体过程为通过 GJR-GARCH 模型拟合残差 序列, 得到沪深综指的条件波动率序 列，换算成年收益波动率，分别得到 0.2613 和 0.2759 。设定沪深综指指数 一点为 100 元人民币和协定价格分别为 2548.99 和 1100.56 。所有计算程序均通 过 Matlab7.10 编程和@RISK 软件完成。

表1 上证综指和深证综指收益率的描述统计特征

\begin{tabular}{|l|l|l|}
\hline & SSCI & SZCI \\
\hline ADF & $-59.2(0.0001)$ & $-26.5(0.0001)$ \\
\hline KPSS & 0.1517 & 0.1979 \\
\hline 均值 & 0.000424 & 0.000642 \\
\hline 标准差 & 0.0182 & 0.0196 \\
\hline 偏度 & -0.2279 & -0.4441 \\
\hline 峰度 & 7.38006 & 6.78599 \\
\hline$J-B$ & $2881.4(0)$ & $2246.9(0)$ \\
\hline$Q^{(36)}$ & $108.8(0)$ & $112.6(0)$ \\
\hline$Q^{2}(36)$ & $1003.9(0)$ & $944.32(0)$ \\
\hline
\end{tabular}

注：SSCI 和 SZCI 收益率序列的 ADF 检验 $1 \%$ 为 -3.431998 ; 5\%为-2.862153. 圆括号内为标准误。 KPSS小于 1 。
表1初步统计分析显示: 沪深综指收 益率左偏且具有明显的尖峰厚尾特征, ADF和KPSS检验表明收益序列0阶平稳, $\mathrm{J}-\mathrm{B}$ 检验说明收益率显著异于正态分 布, Ljung-Box统计量 $Q(k)$ 显示收益率 存在弱自相关, $Q^{2}(k)$ 检验表明收益率的 平方自相关性较强, 序列具有条件异方 差性，可用GJR模型建模。以深证综指 为例, 平方收益序列的自相关见图1。

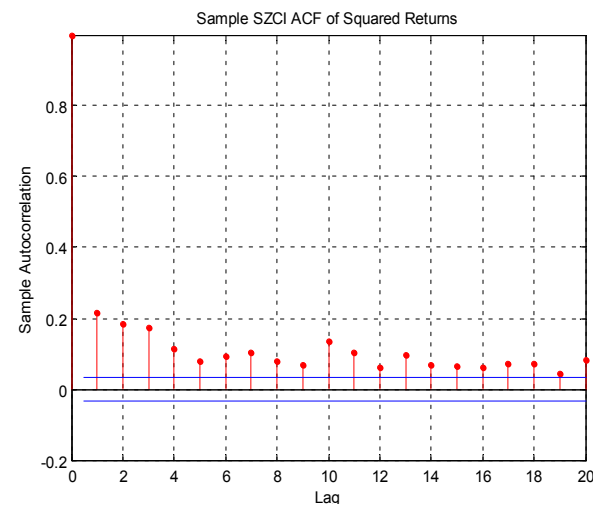

图 1 深证综指的平方收益自相关图

由图1可以看出, 深证综指存在条 件异方差，指数收益序列具有波动集聚 性, 因此运用GJR-GARCH模型拟合数据 的误差项是合理的, 然后通过极大使然 估计得到模型的相应参数。

表 2 AR-GJR-GARCH 模型参数估计结果

\begin{tabular}{|c|c|c|c|c|}
\hline & \multicolumn{2}{|c|}{ 上证综指 } & \multicolumn{2}{|l|}{ 深证综指 } \\
\hline$\not H$ & \multicolumn{2}{|l|}{0.0042} & \multicolumn{2}{|l|}{0.0016} \\
\hline$\omega$ & $\begin{array}{l}4.18 \mathrm{E}-0 \\
6 *\end{array}$ & $\begin{array}{l}-5.20 \mathrm{E}- \\
07\end{array}$ & $\begin{array}{l}5.55 \mathrm{E}-0 \\
6 *\end{array}$ & $\begin{array}{l}-6.50 \mathrm{E}- \\
07\end{array}$ \\
\hline$\alpha$ & $0.0797 *$ & -0.0059 & 0.0991 & -0.0071 \\
\hline$\beta$ & $0.8958 *$ & -0.0037 & \multicolumn{2}{|c|}{$0.8779 *(0.0039)$} \\
\hline$y$ & $0.0347 *$ & -0.0087 & $0.0290 *$ & -0.0103 \\
\hline 对数使然值 & \multicolumn{2}{|l|}{9661.7} & \multicolumn{2}{|l|}{9458.9} \\
\hline K-S 正态检验 & 0.0791 & [0] & 0.0769 & [0] \\
\hline
\end{tabular}

注: () 为标准误, []为概率值. *表示在 0.05 水 平下显著.

由表 2 可知, AR-GJR-GARCH 模型的 参数估计是显著的, $\gamma>0$ 表明沪深市场 
存在非对称杜杆效应, $\alpha+\beta<1$ 说明波 动具有持续性。通过 $\mathrm{S}-\mathrm{plus}$ 软件对沪 深综指进行非参数正态性检验, $\mathrm{K}-\mathrm{S}$ 统 计量相伴概率均为 0 , 表明沪深综指收 益率的非正态性。根据正态分布假定计 算沪深综指期权将很可能会低估期权 面临的市场风险, 因此选用 GJR-GARCH 模型来刻画沪深综指的高峰厚尾和非 对称特征是合适的。

表 3 在不同数值模型下不支付红利欧式期权价格 的比较

\begin{tabular}{|c|c|c|c|c|c|}
\hline & & \multicolumn{2}{|c|}{ 上证综指 (SSCI) } & \multicolumn{2}{|c|}{ 深证综指 (SSCI) } \\
\hline 数值模型 & 模拟次数 & 5000 & 5000000 & 5000 & 5000000 \\
\hline \multirow{2}{*}{$\begin{array}{c}\text { 二叉树模 } \\
\text { 型 }\end{array}$} & 看涨期权 & 227.6195 & & 107.0631 & \\
\hline & 看跌期权 & 72.0914 & & 31.6094 & \\
\hline \multirow{2}{*}{$\begin{array}{c}\text { 三叉树模 } \\
\text { 型 }\end{array}$} & 看涨期权 & 227.6166 & & 107.0598 & \\
\hline & 看跌期权 & 72.0885 & & 31.606 & \\
\hline \multirow{3}{*}{$\begin{array}{c}\text { 蒙特卡洛 } \\
\text { 模拟方法 } \\
\text { 模型 }\end{array}$} & 看涨期权 & 231.404 & 227.4728 & 106.8662 & 107.038 \\
\hline & 看跌期权 & 68.9968 & 72.0697 & 30.7426 & 31.5898 \\
\hline & $\begin{array}{c}\text { 看跌期权 } \\
\text { 95\%置信区 } \\
\text { 间 }\end{array}$ & $\begin{array}{l}{[66.9337,} \\
74.2490]\end{array}$ & $\left|\begin{array}{l}{[72.0877} \\
72.3247]\end{array}\right|$ & $\begin{array}{c}{[29.4359,32} \\
.8345]\end{array}$ & \begin{tabular}{|c}
{$[31.5148$} \\
, 31.6204 \\
]
\end{tabular} \\
\hline \multirow{2}{*}{ B-S 模型 } & 看涨期权 & \multicolumn{2}{|c|}{226.3771} & \multicolumn{2}{|c|}{107.0605} \\
\hline & 看跌期权 & \multicolumn{2}{|c|}{72.7493} & \multicolumn{2}{|c|}{31.6068} \\
\hline
\end{tabular}

基于上述分析, 由表 3 可知, 利用 B-S模型得到 2010年9月 13 日的沪深综 指欧式期权的价格, 再分别通过叉树图 模型和蒙特卡洛模拟对前瞻性的沪深 综指期权重新定价，并与 $B-S$ 模型得到 的价格进行了对比，显然三叉树模型得 到的期权价格更为接近B-S模型的真实 值。作为定价和风险测度的蒙特卡洛在 模拟情景次数达到 500 万次时，也具有 很高的精确度，再结合抽样技术，则在 度量股指期权的市场风险时能得出更 为可靠的结论。

最后, 以上证综指为例, 在持有上 证综指标的多头资产的前提下，基于得
到的欧式看跌期权价格，构建两个组 合：即持有上证综指的多头资产和购买 股指的看跌期权的组合 $\mathrm{A}$ 和仅持有上证 综指的多头资产组合 $\mathrm{B}$ 。然后对组合 $A$ 和 组合B的收益进行对比, 采用@RISK软件 的蒙特卡洛和拉丁方抽样技术, 分别抽 样 1000 和 10000 次得到相应的分位数, 对沪深综指期权的风险进行度量。

表4 基于沪深综指欧式看跌期权价格度量的风险

\begin{tabular}{|c|c|c|c|c|c|c|}
\hline & & $\begin{array}{c}\text { 上证 } \\
\text { 综指 } \\
(\%)\end{array}$ & $\begin{array}{c}\text { 深证 } \\
\text { 综指 } \\
(\%) \\
\end{array}$ & & $\begin{array}{c}\text { 上证 } \\
\text { 综指 } \\
(\%)\end{array}$ & $\begin{array}{c}\text { 深证 } \\
\text { 综指 } \\
(\%) \\
\end{array}$ \\
\hline 抽样 & 统计 & 最小 & 最小 & 抽样 & 最小 & 最小 \\
\hline 技术 & 特征 & 值 & 值 & 技术 & 值 & 值 \\
\hline \multirow{3}{*}{$\begin{array}{c}\text { 蒙特 } \\
\text { 卡洛 } \\
1000\end{array}$} & 收益 & -30 & -29. & \multirow{3}{*}{$\begin{array}{c}\text { 蒙特 } \\
\text { 卡洛 } \\
1000 \\
0\end{array}$} & -35 & -34 \\
\hline & 1 & 0 & 8 & & 3 & 4 \\
\hline & $\begin{array}{c}\text { 收益 } \\
2\end{array}$ & -7.7 & -8.3 & & -7.7 & -8.3 \\
\hline \multirow{4}{*}{$\begin{array}{c}\text { 拉丁 } \\
\text { 方 } \\
1000\end{array}$} & 收益 & -32 & -33 & \multirow{3}{*}{$\begin{array}{c}\text { 拉丁 } \\
\text { 方 } \\
1000 \\
0\end{array}$} & -37 & -38 \\
\hline & 3 & 7 & 4 & & 3 & 7 \\
\hline & $\begin{array}{c}\text { 收益 } \\
4\end{array}$ & -7.7 & -8.3 & & -7.7 & -8.3 \\
\hline & $\begin{array}{c}\text { 置信 } \\
\text { 度 }\end{array}$ & 95 & 95 & $\begin{array}{c}\text { 置信 } \\
\text { 度 }\end{array}$ & 95 & 95 \\
\hline \multirow{2}{*}{$\begin{array}{c}\text { 蒙特 } \\
\text { 卡洛 } \\
1000\end{array}$} & VaR1 & $\begin{array}{c}-14 . \\
9\end{array}$ & $\begin{array}{c}-16 . \\
3\end{array}$ & \multirow{2}{*}{$\begin{array}{c}\text { 蒙特 } \\
\text { 卡洛 } \\
1000 \\
0 \\
\end{array}$} & $\begin{array}{c}-14 . \\
3\end{array}$ & $\begin{array}{c}-15 . \\
2\end{array}$ \\
\hline & VaR2 & -7.7 & -8.3 & & -7.7 & -8.3 \\
\hline \multirow{2}{*}{$\begin{array}{c}\text { 拉丁 } \\
\text { 方 } \\
1000\end{array}$} & VaR3 & $\begin{array}{c}-14 \\
5\end{array}$ & $\begin{array}{c}-15 . \\
6\end{array}$ & \multirow{2}{*}{$\begin{array}{c}\text { 拉丁 } \\
\text { 方 } \\
1000 \\
0 \\
\end{array}$} & $\begin{array}{c}-14 . \\
4\end{array}$ & $\begin{array}{c}-15 . \\
6\end{array}$ \\
\hline & VaR4 & -7.7 & -8.3 & & -7.7 & -8.3 \\
\hline
\end{tabular}

由表4可知，基于抽样技术的收敛 性，拉丁方抽样技术在 1000 次小样本 抽样中，更快的收敛于真实分布，其 收益和VaR值更为平稳和精确。蒙特卡 洛抽样技术，进行10000次抽样模拟， 收敛性较好，其收益和 $\mathrm{VaR}$ 值更为精 确。同时，在有无购买欧式看跌期权 的组合 $A$ 和 $B$ 的收益对比中, 以上证综 指拉丁方 1000 次抽样为例, 若无购买, 在置信度 $95 \%$ 下的 VaR 则为上证综指 现值的 $-14.5 \%$, 即 VaR 为-389.8点, 按 
设定每点 100 元计损失为 38980 元; 若 购买, 在置信度 $95 \%$ 下的 VaR则相应下 降为 $-7.7 \%$, 即 $V a R$ 为 -207 点, 损失为 20700 元。同时还注意到，若没有购买 欧式看跌期权，则上证综指还存在最 大损失为 $-32.7 \%$ 的可能, 即 $\mathrm{VaR}$ 为 -879 点, 最大损失为 87900 元。而购买看跌 期权其最大损失仍为 20700 元，显然， 购买看跌期权的组合 $\mathrm{A}$ 锁定风险较好。

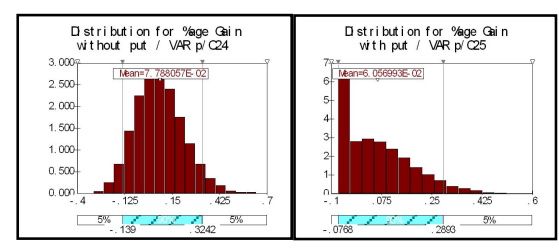

图 2 有无购买欧式看跌期权的上证综指组合收

\section{益分布对比}

从图2可知，图示为上证综指以拉 丁方抽样 1000 次为例, 组合 $A$ 和B的收益 分布，若没有购买欧式看跌期权，组合 $\mathrm{B}$ 存在损失其价值为 $-32.7 \%$ 的可能; 若 购买欧式看跌期权，则组合 $\mathrm{A}$ 的平均收 益仅为 $6.056 \%$, 与组合 $\mathrm{B}$ 相比, 平均收 益减少 $1.73 \%$, 即收益减少 4650 元。显 然购买欧式看跌期权更像是为规避和 控制风险付出的相应成本。

\section{4.结论}

通过计算和分析，我们可以得到如 下结论: (1)基于拉丁方抽样技术, 在较 小样本得到的沪深综指收益和 $\mathrm{VaR}$ 值比 采用基于蒙特卡洛抽样得到的更为平 稳和精确, 但在较大样本抽样中则刚好 相反，基于蒙特卡洛抽样技术相对较 优; (2)在基于欧式看跌期权的风险度量 中, 以上证综指为例中持有多头资产组 合和购买欧式看跌期权的组合可能损
失仅为 $-7.7 \%$, 而无购买欧式看跌期权 的资产组合则存在损失其价值为 $-32.7 \%$ 的可能性, 同时前者还大幅降低了VaR, 提高了投资现金流的比例，显然购买欧 式看跌期权虽付出了相应成本，但可以 更好地规避和控制持有多头资产组合 大幅损失的风险。

\section{参考文献}

[1] Cormac Butler.于研,刘丹丹,陈勇译. 风险值概论.[M].上海财经大学出 版社.2002:68-96.

[2] PietroPenza,VipulK.Bansal.Measurin $g$ Market Risk with value at Risk[M].JohnWiley\&Sons,2001:88167.

[3] 杨春鹏,崔援民. 非对称金融衍生工 具的风险度量指标确 $V a R$ 研究 $[\mathrm{J}]$. 预测.1999,(4):39-43.

[4] 张勇,王建稳,英英.期权风险的 VaR 度量研究. 北方工业大学学 报.2005,1:77-79.

[5] J.Cox and S.Ross. The valuation of options for alternative stochastic process[J].Journal of Financial Economies.1976,(3):45-166.

[6] J.C.Cox,S.A.Ross,M.Rubinstein. Option Pricing:a simplified approach[J].Journal Financial Economics.1979,(7):229-263.

[7] 金龙,王正林.精通MATLAB 金融计 算 $[\mathrm{M}]$. 北京：电子工业出版 社.2009:223-224. 